\title{
An Improved Hybrid Algorithm Based on PSO and BP for Stock Price Forecasting
}

\author{
Ying Sun ${ }^{1,2}$ and Yuelin Gao ${ }^{1,2, *}$ \\ ${ }^{1}$ School of Computer and Information, Hefei University of Technology, Hefei, Anhui, 230009, China; ${ }^{2}$ Institute of \\ Information \& System Science, Beifang University of Nationalities, Yinchuan, Ningxia, 750021, China
}

\begin{abstract}
Stock price prediction is the main concern for financial firms and private investors. In this paper, we proposed a hybrid BP neural network combining adaptive PSO algorithm (HBP-PSO) to predict the stock price. HBP-PSO takes full use of the global searching capability of PSO and the local searching advantages of BP Neural Network. The PSO algorithm is applied for training the connection weights and thresholds of BP, in order to take advantage of BP, each particle in PSO swarm will be optimized by error correcting method of BP in probability. The trained BP neural network is used to predict the stock price. The empirical analysis using the real data of Chinese stock market demonstrates the feasibility and effectiveness of this method.
\end{abstract}

Keywords: Adaptive PSO, BP neural network, stock price forecasting.

\section{INTRODUCTION}

The stock market is a vital part of financial market and the basis and core of the capital market. It is the important mechanism of resource allocation in market economy, and has great role in promoting economic development. Stock price forecasting is an important research subject that has attracted researchers' attention. The accuracy of stock price forecasting is very difficult, because it is usually influenced by many factors, such as general economic conditions, political environments and investor behaviors. How to adopt reasonable methods and models to forecast the change trend of stock price is a big focus in the academic world. At present stage, the research of stock price forecasting mainly concentrate in two aspects: one is the traditional volatility forecasting model based on statistical principle, which mainly are ARCH models [1] and SV model [2]. But because these models are based on the theory of statistical analysis, they have higher requirements on the sample data for forecasting. In fact, even if there are a large sample data, but it does not necessarily find rule, even if there is a statistical rule, but it is also not a typical. The other is the artificial intelligence technique such as artificial neural network (ANN) [3-5], genetic algorithm (GA)[6,7], and many hybrid intelligent algorithms [8-11]. The hybrid intelligent algorithms have more flexibility to solve the complex models, so more and more researchers tend to use them to deal with forecasting problems.

Therefore, this paper presents a BP Neural Network combining adaptive PSO algorithm (HBP-PSO algorithm) to forecast the stock price trend, which organically mixes the global searching ability of PSO and local optimal of BP Neural Network. This new model can greatly improve the accuracy of the change tendency forecast and that could provide a forecasting method to investors.

The rest of the paper is organized as follows. In section 2, we describe the sketch of standard PSO algorithm. Thereafter, in section 3, we present a BP Neural Network combining adaptive PSO algorithm to forecast the price of stock. The empirical research and simulation are provided in section 4 . The paper concludes in section 5 .

\section{PARTICLES SWARM OPTIMIZATION ALGO- RITHM}

PSO was presented by Kennedy and Eberhart in 1995 [12]. It is a random optimization algorithm based on swarm aptitude. Its thought comes from the research on the behavior of the bird swarm catching food. PSO is an iterative optimization tool, which randomly generate a group of initial solutions and through the iterative search to find the optimal value.

Let $D$ be the dimension of the search space, $x_{i}=\left(x_{i 1}, x_{i 2}, \cdots, x_{i D}\right)$ be the current position of the $i^{\text {th }}$ particle in swarm, $P_{i}=\left(p_{i 1}, p_{i 2}, \cdots, p_{i D}\right)$ be the best position of the $i^{\text {th }}$ particle so far, and $p_{g}=\left(p_{g^{1}}, p_{g^{2}}, \cdots, p_{g^{D}}\right)$ be the best position which of the whole swarm have ever visited. The rate of the velocity for the $i^{\text {th }}$ particle is noted as $v_{i}=\left(v_{i 1}, v_{i 2}, \cdots v_{i D}\right)$.

\section{Algorithm 1 Standard Particle Swarm Optimization}

Step 1: Initialize a population of particles $X_{m}$ with random position vector $x_{i}$ and the velocity vector $v_{i}$, setting parameters $c_{1}$ and $c_{2}$, the maximum generation $T_{\max }$, the generation number $T:=0$; 
Step 2: Calculate the fitness of all the particles in $\mathrm{X}(T)$;

Step 3: Renew the position and velocities of particles based on equation:

$v_{i d}^{T+1}=w v_{i d}^{T}+c_{1} r_{1}\left(p_{i d b e s t} x_{i d}^{T}\right)+c_{2} r_{2}\left(g_{\text {id best }}-x_{i d}^{T}\right)$

$x_{i d}^{T+1}=x_{i d}^{T}+v_{i d}^{T+1}$

Step 4: Calculate the particle's fitness and renew every particle's optimal position and the global optimal position.

Step 5: (Termination examination) If the termination criterion is satisfied, then output he global optimal position and its fitness value. Otherwise, let $T:=T+1$, and Loop to step2.

\section{THE DESCRIPTION OF HNP-PSO ALGORITHM}

The BP neural network [13] the error back propagation neural network, which is a kind of supervised learning neural network. In BP neural network, the signal is inputted from outside spreads to the output layer. If the output value is not the expected output, it shifts to the conversed spreading processing and the error (the true value- the real output value) is reduced by modifying contacted weight value of neurons in every layer. Then it shifts to the positive spreading processing and revolves iteration until the error is smaller than the given value. However, the BP neural network often fall into local minimum point in its learning process, thus it can't search to the global optimal value. PSO algorithm is an intelligent optimization method, which adopt the global random searching method to find the optimal value. But its local search ability is not strong. The HBP-PSO algorithm gives full play to the advantages of two algorithms and improves the performance of the new algorithm.

The process of this hybrid algorithm is as below:

(1) Initialization neural network. With the basic principle of neural network, a topology structure of neural network is built based on the sample set of input and output. All the connection weights and thresholds are encoded into real vector, which are individual particles in the population. The dimension of the particle position and velocity vector is $D$, which is the number of connection weights and thresholds.

(2) Initialization PSO algorithm. Assume that population size $m$, let $x_{i}=\left(x_{i 1}, x_{i 2}, \cdots, x_{i D}\right)$ be the current position of the $i^{\text {th }}$ particle in swarm, which is expressed connection weights and thresholds, setting parameters $c_{1}, c_{2}$ and adaptive inertia weight $w=w_{i n i}+\frac{T\left(w_{\text {end }}-w_{i n i}\right)}{T_{\max }}, w_{i n i}$ is the initial value of the adaptive inertia weight, $w_{\text {end }}$ is the final value of the adaptive inertia weight, $T$ is the generation number, $T_{\max }$ is the maximum generation number, the rate of the velocity for the $i^{\text {th }}$ particle is noted as $v_{i}=\left(v_{i 1}, v_{i 2}, \cdots v_{i D}\right)$.Set the termination criterion. Randomly generate the initial population $\mathrm{X}(0) . T:=0$.
(3) Calculate the fitness of all the particles. Based on the theory of signals forward propagation of BP Neural Network, we can calculate the actual output of each particle by formula $O_{p}=F_{l}\left(\ldots\left(F_{2}\left(F_{1}\left(X_{p} W^{(1)}\right) W^{(2)}\right) \ldots\right) W^{(L)}\right)$. Comparing $O_{p}$ with the ideal output, we can calculate the error of $p$ sample and then get the error of all sample data. Here, we use the mean value of the error of all sample data as a fitness value of each particle.

$$
\begin{aligned}
& E_{i}=\sum_{j}\left(\mathrm{y}_{i j}-o_{i j}\right)^{2} \\
& \bar{E}_{l}=\frac{1}{n} \sum_{i=1}^{n} n e t_{i}
\end{aligned}
$$

Here $L$ is the layer number of neural network, $F\left({ }^{*}\right)$ is called the activation function of each layer, $X_{p}$ is the input sample, $W$ is connection weights, $n$ is the number of the sample set. $y_{i}$ is the idea output of the $i^{\text {th }}$ sample, $o_{i}$ is the actual output of the $i^{t h}$ sample. $E_{i}$ is the error value of the $i^{\text {th }}$ sample, $E_{i}$ is the fitness value of the $l^{\text {th }}$ particle (that is, the mean value of the error of all sample data).

(4) Renew the velocity and position of particles based on equation (1) and (2).

(5) Set the probability $P$

$P=\frac{T}{T_{\max }}$

Obviously, this formula is an increased with the increase of the iteration number. Randomly generated a random number rand $\in[0,1]$, when $\operatorname{rand}<P$, the connection weights and thresholds are adjusted through the operation principle of BP neural network. The error correction formula as follow:

Output layer:

$$
\begin{aligned}
w_{p q} & =w_{p q}+\Delta w_{p q} \\
\Delta w_{p q} & =\alpha \delta_{q} o_{p} \\
& =\alpha o_{p}\left(1-o_{q}\right)\left(\mathrm{y}_{q}-o_{q}\right) o_{p}
\end{aligned}
$$

Here $w_{p q}$ is the connection weight between $q^{\text {th }}$ neuron in output layer and $p^{\text {th }}$ neuron in its leading layer. $\alpha$ is learning rate.

Hidden layer:

$$
\begin{aligned}
v_{h p}= & v_{h p}+\Delta v_{h p} \\
\Delta v_{h p}= & \alpha \delta_{p k-1} o_{h k-2} \\
= & \alpha o_{p k-1}\left(1-o_{p k-1}\right)\left(w_{p 1} \delta_{1 k}+w_{p 2} \delta_{2 k}\right. \\
& \left.+\ldots+w_{p m} \delta_{m k}\right) o_{h k-2}
\end{aligned}
$$

Here $v_{h p}$ is the connection weight between $h^{\text {th }}$ neuron in $(k-2)^{t h}$ hidden layer and $p^{\text {th }}$ neuron in $(k-1)^{t h}$ hidden layer, $\alpha$ is learning rate, $o_{p k-1}$ and $o_{h k-2}$ are the actual output, $\mathrm{y}_{q}$ is the idea output, $w_{p 1}, w_{p 2}, \cdots w_{p m}$ are weight val- 
ues, $\delta_{1 k}, \delta_{2 k} \ldots \delta_{m k}$ are errors between the $p^{t h}$ neuron in $(k-1)^{t h}$ hidden layer and each neuron in $k^{\text {th }}$ hidden layer.

The reason for this is that the PSO algorithm has better global search ability for optimizing the weight and threshold, so the PSO algorithm is first used to globally search, which can overcome the problem of BP neural network easily trapping in local optimum. The local searching ability of BP neural network primarily to be used in the latter period, the probability of running becomes bigger as the increase of iteration times.

(6) Calculate the particle's fitness and renew every particle's optimal position $p_{i}, i=1, \cdots, m$ and the global optimal position $p_{g}$.

(7) Termination examination. If the termination criterion is satisfied, then output the global optimal position and its fitness value. Otherwise, let $T:=T+1$, and Loop to (3). The flow chart of HBP-PSO algorithm is depicted in Fig. (1).

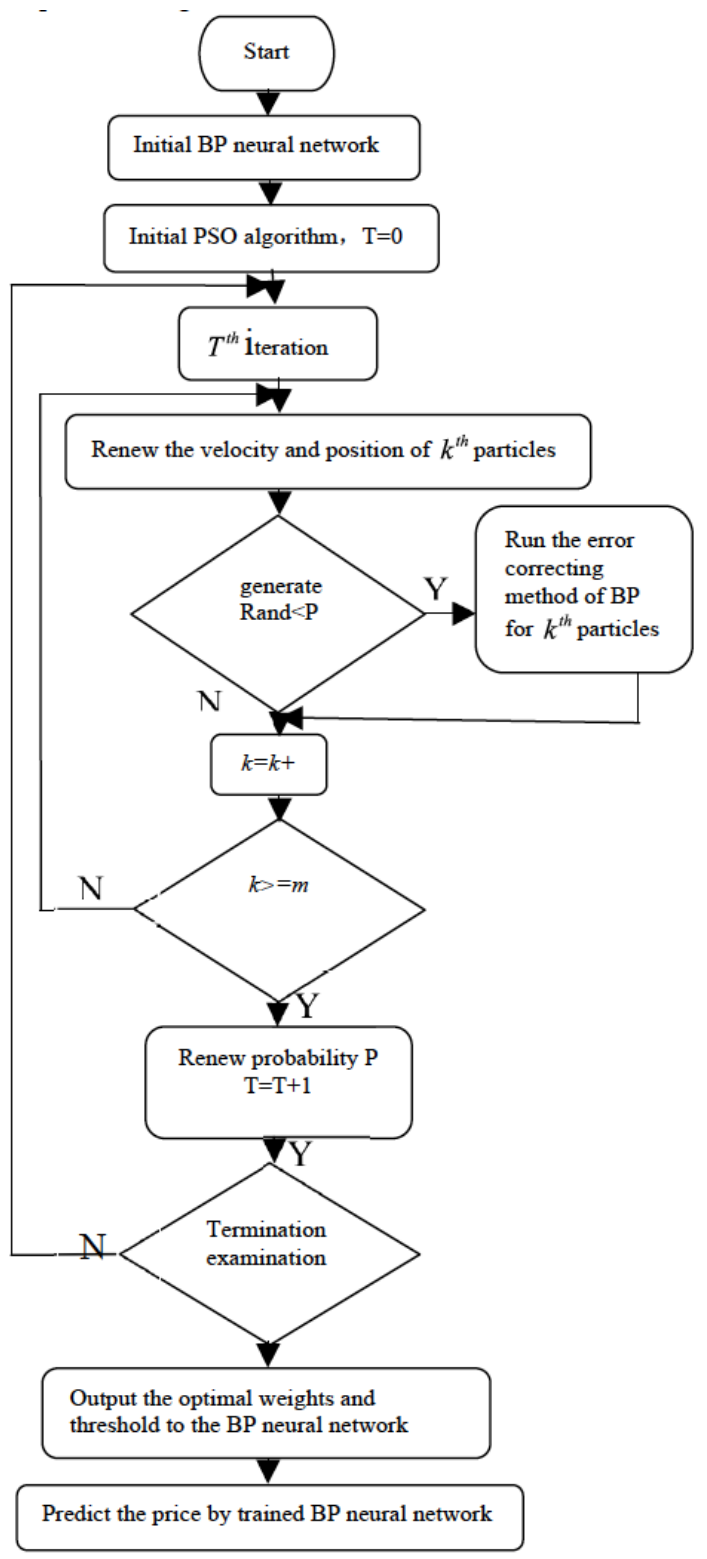

Fig. (1). The flow chart of HBP-PSO.

\section{SIMULATION EXPERIMENTS}

\subsection{Experimental Data}

In this section we implement the proposed HBP-PSO algorithm using the daily stock price of "Zhong Guo Yi Yao"(600056), which was launched in Shanghai Stock Exchange, China. The historic data covers the financial timeseries data form $2009 / 11 / 18$ to $2010 / 3 / 15$, total 75 group data sample. Top 60 group data is used for training and the last 15 group data is used for testing. In this model, 15 parameters, which are opening price, closing price, ceiling price, the lowest price, volume of three days, as the net input data, and the Price Change Ratio three days later as the output data.

\subsection{Parameter Setting}

The HBP-PSO network has 3 layers, which is composed of 15 input neurons (input parameters is 15 ), 8 hidden neurons (experiments show the number bigger than 8 , the volatility higher) and 1 output neurons (only one output parameter). The target error goal is 0.0001 , learning rate $\alpha=0.5$.

The population size of PSO algorithm is 40 , social coefficient $c_{1}=0.5$, cognitive coefficient $c_{2}=0.5$, the initial value of the adaptive inertia weight $w_{i n i}=0.9$, the final value $w_{\text {end }}=0.4$, the maximum generation number $T_{\text {max }}=200$.

\subsection{Performance Analysis}

For this stock, the trend prediction correct rate of HBPPSO is about $70 \%$, and the standard BP model is about $50 \%$. Obviously, the predict accuracy of HBP-PSO is better than standard BP model. The forecasting results of the stock are shown in Figs. (2-4), respectively.

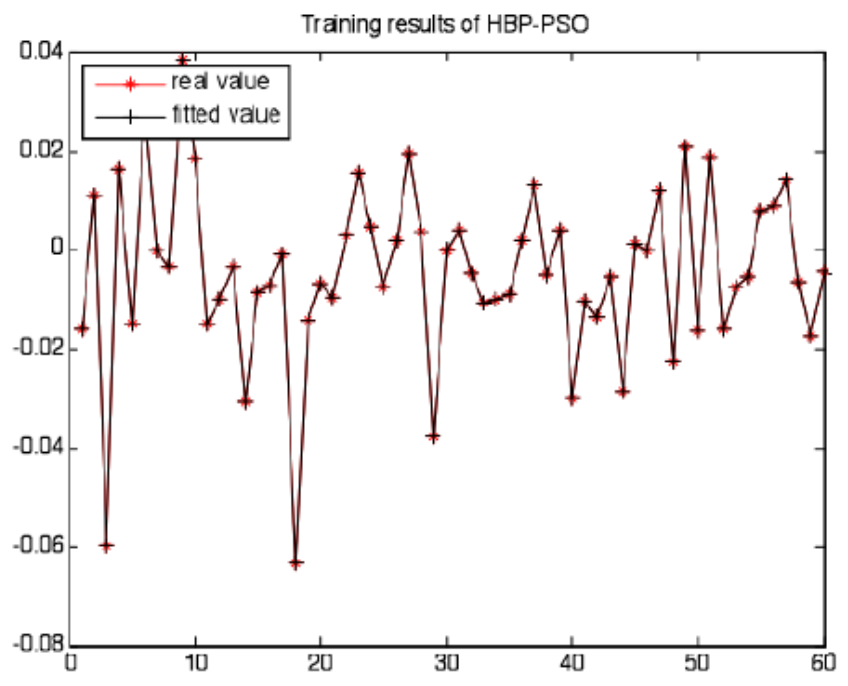

Fig. (2). The fitted value of the price change ratio of training data.

The program run result shows that although the actual value and predicted value are not identical, but the error is small, the result is acceptable. Specifically, we can see that HBP-PSO predicting trend is basic agreement with the real value, which can give investors some useful information to 


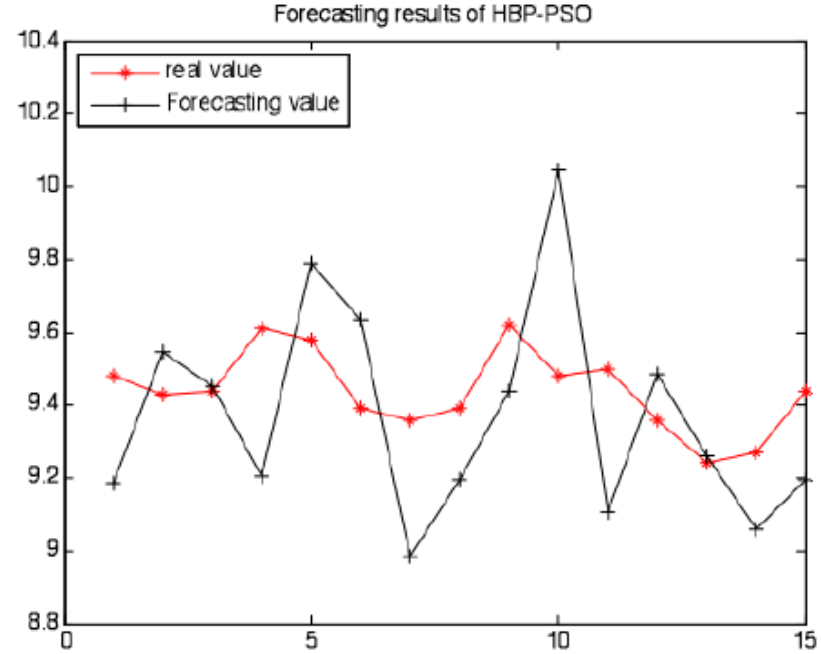

Fig. (3). The forecasting value of closing price of testing data.

adjust the stock trading. So the HBP-PSO algorithm has good prediction effect on stock price, and its network parameter converges fast and no oscillation. Of course, if new elements are added to the network learning process, the stock price accuracy can improve, but the difficulty of the network will increase. In fact, most investors are concerned about the trend of changes in future price of stock market, not the exact value. Therefore, The HBP-PSO algorithm predicts the trend of stock price is practical.

\section{CONCLUSION}

The stock market is a complex nonlinear dynamic system, but the neural network has powerful nonlinear mapping ability, which can achieve any complicated causality. This paper presents a hybrid neural network---HBP-PSO, which has good application in the stock price trend forecast. Because of the particularity of the stock market, it will be affected by the national policy, macroeconomic, etc. If more reasonable factors can be quantified, the forecast accuracy of stock price volatility will be greatly improved.

\section{CONFLICT OF INTEREST}

The authors confirm that this article content has no conflict of interest.

\section{ACKNOWLEDGEMENTS}

This work is supported by the National Natural Science Foundation of P.R. China (60962006), the Foundations of research projects of State Ethnic Affairs Commission of P.R. China (14BFZ003) and the Teaching reform project of Ning Xia Higher Education (2012).

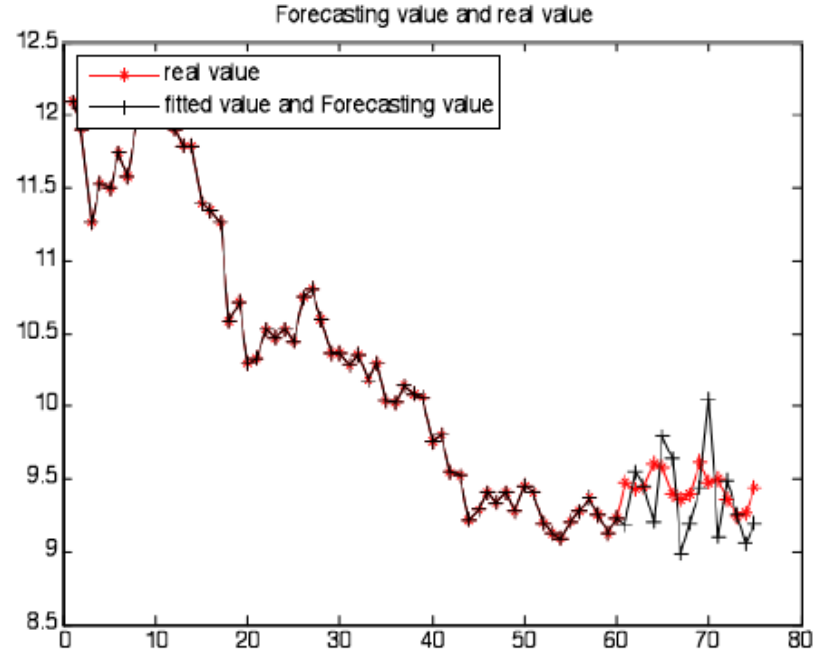

Fig. (4). The fitted and forecasting value of closing price of all sample data.

\section{REFERENCES}

[1] R. F. Engle, "Autoregressive conditional heteroscedasticity with estimates of the variance of United Kingdom inflation.," Econometrica, vol. 50, pp. 987-1007, 1982.

[2] E. Ghysel, A. C. Harvey, and E. Renault, "Stochastic volatility," Review of Economic Studies, vol. 1994, pp.132- 183, 1994.

[3] L. Huo, B. Jiang, T. Ning, and B. Yin, "A BP neural network predictor model for stock price," Intelligent Computing Methodologies, vol. 8589, pp. 362-368, 2014.

[4] Z. Chengzhao, P. Heiping, and Z. Ke, "Comparison of back propagation neural networks and EMD-Based neural networks in forecasting the three major Asian stock markets," Journal of Applied Sciences, vol. 15, no. 5, pp.731-754, 2015.

[5] J. L. Ticknor, "A Bayesian regularized artificial neural network for stock market forecasting," Expert Systems with Applications, vol. 40, no.14, pp. 5501-5506, 2013.

[6] Y. Sun, X. Li, C. Liu, and J. Wang, "Genetic algorithm based markowitz portfolio model and stock price behavior," Journal of Systems \& Management, vol. 19, pp. 84-90, 2012.

[7] K. Yang, M. Wu, and J. Lin, "The application of fuzzy neural networks in stock price forecasting based On Genetic Algorithm discovering fuzzy rules," Natural Computation (ICNC), $8^{\text {th }}$ International Conference, pp. 470-474, 2012.

[8] J. Sen, and A. K. Das, "Artificial neural network model for forecasting the stock price of Indian IT company," In: Proceedings of the Second International Conference on Soft Computing for Problem Solving (SocProS 2012), vol.12, pp. 28-30, 2012.

[9] P. Chang, D. Wang, and C. Zhou, "A novel model by evolving partially connected neural network for stock price trend forecasting", Expert Systems with Applications, vol. 39, no.1, pp. 611-620.

[10] A.A. Adebiyi, A.O. Adewumi, and C.K. Ayo, "Comparison of ARIMA and artificial neural networks models for stock price prediction", Journal of Applied Mathematics, vol. 33, no.1, pp. 75-81, 2014.

[11] S. Hsu, J. Hsieh, T. Chih, K.Hsu, "A two-stage architecture for stock price forecasting by integrating self-organizing map and support vector regression," Expert Systems with Applications, vol. 36, no. 4, pp. 7947-7951, 2009.

[12] J. Kennedy, and R. Eberhart, "Particle swarm optimization," Neural Networks, Proceedings on IEEE International Conference, pp. 1942-1948, 1995.

[13] X. Xiong, and M. Vie, "BP Neural Network principle and MATLAB Simulation," [http://paper.edu.cn.]. 